International Journal of Pure and Applied Mathematics

Volume 85 No. 4 2013, 761-770

ISSN: 1311-8080 (printed version); ISSN: 1314-3395 (on-line version)

url: http://www.ijpam.eu

doi: http://dx.doi.org/10.12732/ijpam.v85i4.12

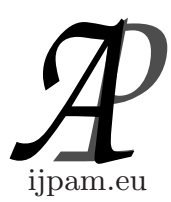

\title{
MATCHING AND EDGE COVERING NUMBER ON TENSOR PRODUCT OF FAN GRAPH
}

\author{
Siriluk Intaja ${ }^{1}$, Thanin Sitthiwirattham ${ }^{2} \S$ \\ ${ }^{1,2}$ Department of Mathematics \\ Faculty of Applied Science \\ King Mongkut's University of Technology \\ North Bangkok, Bangkok, 10800, THAILAND \\ ${ }^{2}$ Centre of Excellence in Mathematics, CHE \\ Sri Ayutthaya Road, Bangkok, 10400, THAILAND
}

\begin{abstract}
Let $\alpha^{\prime}(G)$ and $\beta^{\prime}(G)$ be the matching number and edge covering number of $G$, respectively. The tensor product $G_{1} \otimes G_{2}$ of graph of $G_{1}$ and $G_{2}$ has vertex set $V\left(G_{1} \otimes G_{2}\right)=V\left(G_{1}\right) \times V\left(G_{2}\right)$ and edge set $E\left(G_{1} \otimes G_{2}\right)=$ $\left\{\left(u_{1} v_{1}\right)\left(u_{2} v_{2}\right) \mid u_{1} u_{2} \in E\left(G_{1}\right)\right.$ and $\left.v_{1} v_{2} \in E\left(G_{2}\right)\right\}$. In this paper, we determined generalization of matching number and edge covering on tensor product of fan graph and any simple graph.
\end{abstract}

AMS Subject Classification: 05C69, 05C70, 05C76

Key Words: tensor product, matching number, edge covering number

\section{Introduction}

In this paper, graphs must be simple graphs which can be trivial graph. Let $G_{1}$ and $G_{2}$ be graphs. The tensor product of graph $G_{1}$ and $G_{2}$, denote by $G_{1} \otimes G_{2}$, is the graph with $V\left(G_{1} \otimes G_{2}\right)=V\left(G_{1}\right) \times V\left(G_{2}\right)$ and $E\left(G_{1} \otimes G_{2}\right)=$ $\left\{\left(u_{1} v_{1}\right)\left(u_{2} v_{2}\right) \mid u_{1} u_{2} \in E\left(G_{1}\right)\right.$ and $\left.v_{1} v_{2} \in E\left(G_{2}\right)\right\}$.

Next, we give the definitions about some graph parameters.

A subset $M$ of the edge set $E$ of $G$ is said to be a matching or an independent edge set of $G$ if no two distinct edges in $M$ have a common vertex. A matching

Received: February 26, 2013

(c) 2013 Academic Publications, Ltd. url: www.acadpubl.eu

$\S$ Correspondence author 
$M$ is called a maximum matching in $G$ if there is no matching $M^{\prime}$ of $G$ with $\left|M^{\prime}\right|>|M|$. The cardinality of a maximum matching of $G$ is call the matching number of denoted by $\alpha^{\prime}(G)$.

An edge of graph $G$ is said to cover the two vertices incident with it, and an edge cover of a graph $G$ is a set of edges covering all the vertices of $G$. The minimum cardinality of an edge cover of a graph $G$ is called the edge covering number of, $G$ denote by $\beta^{\prime}(G)$.

By definitions of matching number and edge covering number, clearly that

$$
\alpha^{\prime}\left(F_{m, n}\right)= \begin{cases}n, & m \geq n \\ m+\left\lfloor\frac{n-m}{2}\right\rfloor, & m<n\end{cases}
$$

and

$$
\beta^{\prime}\left(F_{m, n}\right)= \begin{cases}m, & m \geq n \\ m+\left\lceil\frac{n-m}{2}\right\rceil, & m<n .\end{cases}
$$

Proposition 1.1 Let $H=G_{1} \otimes G_{2}=(V(H), E(H))$ then:

(i) $|V(H)|=\left|V\left(G_{1}\right)\right|\left|V\left(G_{2}\right)\right|$

(ii) $|E(H)|=2\left|E\left(G_{1}\right)\right|\left|E\left(G_{2}\right)\right|$

(iii) for every $(u, v) \in V(H), d_{H}((u, v))=d_{G_{1}}(u) d_{G_{2}}(v)$.

Theorem 1.2 Let $G_{1}$ and $G_{2}$ be connected graphs, The graph $H=G_{1} \otimes G_{2}$ is connected if and only if $G_{1}$ or $G_{2}$ contains an odd cycle.

Theorem 1.3 Let $G_{1}$ and $G_{2}$ be connected graphs with no odd cycle then $G_{1} \otimes G_{2}$ has exactly two connected components.

Next we get that general from of graph of tensor product of $F_{m, n}$ and a simple graph.

Proposition 1.4 Let $G$ be a connected graph of order $p$, the graph of $F_{m, n} \otimes$ $G$ is

$$
\bigcup_{i=1}^{m} H_{i} \cup \bigcup_{i=m+1}^{m+n-1} M_{i} \quad ; \quad H_{i}=\bigcup_{j=m+1}^{m+n} H_{i j} \quad \text { and } \quad M_{i}=H_{i(i+1)},
$$

where

$$
\begin{gathered}
V\left(H_{i j}\right)=S_{i} \cup S_{j}, S_{i}=\{(i, 1),(i, 2), \ldots,(i, p)\}, \\
S_{j}=\{(j, 1),(j, 2), \ldots,(j, p)\} ; i<j \text { and } E\left(H_{i j}\right)=\{(i, u)(j, v) / u v \in E(G)\} .
\end{gathered}
$$

Moreover, if $G$ has no odd cycle then each $H_{i j}$ has exactly two connected components isomorphic to $G$.

Example 


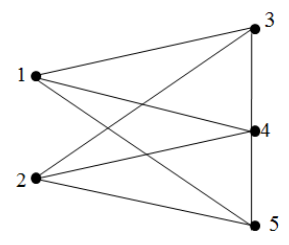

$F_{2,3}$

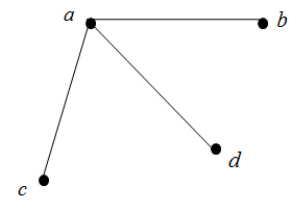

$G$

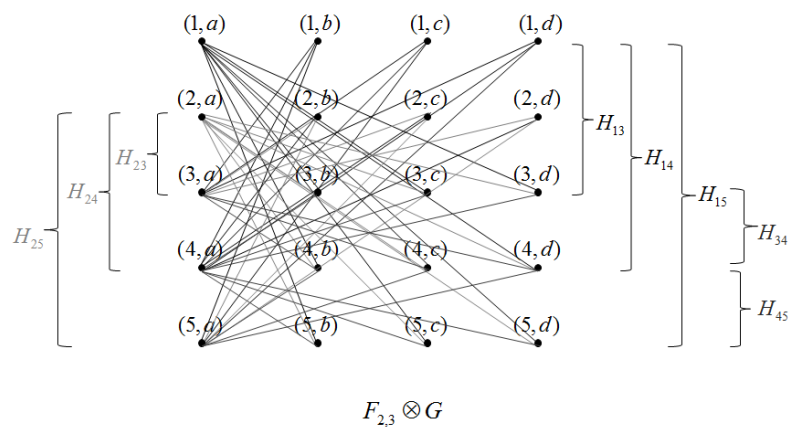

Figure 1: The graph of $F_{m, n} \otimes G$

\section{Matching Number of the Graph of $F_{m, n} \otimes G$}

We begin this section by giving the definition and theorem for alternating path and augmenting path, Lemma 2.3. that show character of matching for each $H_{i j}$.

Definition 2.1. Let Given a matching $M$, an $M$ - alternating path is a path that alternates between edges in $M$ and edges not in $M$. An $M$-alternating odd path whose endpoints are unsaturated by $M$ is an $M$-augmenting path.

Theorem 2.2. A matching $M$ in a graph $G$ is a maximum matching in $G$ if and only if $G$ has no $M$-augmenting path.

Lemma 2.3. Let $F_{m, n} \otimes G=\bigcup_{i=1}^{m} H_{i} \cup \bigcup_{i=m+1}^{m+n-1} M_{i} ; H_{i}=\bigcup_{j=m+1}^{m+n} H_{i j}$ and $M_{i}=H_{i(i+1)}$, then $\alpha^{\prime}\left(H_{i j}\right)=\alpha^{\prime}\left(H_{i(i+1)}\right)=2 \alpha^{\prime}(G)$.

Proof. Suppose $G$ has no odd cycle, by proposition 1.4, we have that $H_{i j}$ and $H_{i(i+1)}$ each contains 2 components isomorphic to $G$. Therefore $H_{i j}=$ $H_{i(i+1)}=2 G$. So $\alpha^{\prime}\left(H_{i j}\right)=\alpha^{\prime}\left(H_{i(i+1)}\right)=2 \alpha^{\prime}(G)$. 
If $G$ has odd cycle, we have $d_{H_{i j}}\left(u_{i}, v\right)=d_{H_{i j}}\left(u_{j}, v\right)=d_{G}(v)$, for $\left(u_{i}, v\right) \in$ $S_{i}$ and $\left(u_{j}, v\right) \in S_{j}, i<j$.

Let $E^{*}=\left\{e_{i} \mid e_{i}\right.$ is any one edge in edge in each odd cycle $C_{i}$ in $G ; i=$ $1,2, \ldots, l\},\left|E^{*}\right| \leq l$ and let $M$ be a maximum matching set of $G$. Now consider the tensor product

$$
\bigcup_{i=1}^{m} H_{i}^{*} \cup \bigcup_{i=m+1}^{m+n-1} H_{i(i+1)}^{*}=F_{m, n} \otimes\left(G-E^{*}\right) ; i<j, i=1,2, \ldots, m \text { where }
$$
$V\left(H_{i j}^{*}\right)=V\left(H_{i j}\right), E\left(H_{i j}^{*}\right)=(i, u)(j, v) \mid u v \in\left(G-E^{*}\right)$. We get $H_{i j}=H_{i(i+1)}=$ $2\left(G-E^{*}\right)$ then

$$
\alpha^{\prime}\left(H_{i j}^{*}\right)=\alpha^{\prime}\left(H_{i(i+1)}^{*}\right)=2 \alpha^{\prime}\left(G-E^{*}\right)= \begin{cases}2\left[\alpha^{\prime}(G)-\left|E^{* *}\right|\right], & \text { if } E^{* *} e_{i} \in M \\ 2 \alpha^{\prime}(G), & \text { otherwise }\end{cases}
$$

When we add $E^{*}$ comeback, we get $\alpha^{\prime}\left(H_{i j}\right)=\alpha^{\prime}\left(H_{i(i+1)}\right)=2\left|E^{* *}\right|$. Hence $\alpha^{\prime}\left(H_{i j}\right)=\alpha^{\prime}\left(H_{i(i+1)}\right)=2^{\prime} \alpha(G)$.

Next, we establish theorem 2.3 for a maximum matching number of $F_{m, n} \otimes$ $G$.

Lemma 2.3 Let $G$ be connected graph order p, then

$$
\alpha^{\prime}\left(F_{m, n} \otimes G\right)= \begin{cases}2 n \alpha^{\prime}(G), & m=n \text { or } m<n \text { and } \\ 2 n \alpha^{\prime}(G)+(m-n)\left|M^{* *}\right|, & m>n \\ 2 m \alpha^{\prime}(G)+\left|M^{* *}\right|, & m<n \text { is perfectmatching } n-m=1 \\ (m+n) \alpha^{\prime}(G), & m<n \text { and } n-m \text { is even } \\ (m+n-1) \alpha^{\prime}(G)+\left|M^{* *}\right|, & m<n \text { and } n-m \text { is odd }\end{cases}
$$

where $M^{* *}=\{u v \in E(G) \mid u$ is not matched in maximum matching in $G$ and $\left.v \in N_{G}(u)\right\}$.

Proof. Let $V\left(\bar{K}_{m}\right)=\left\{u_{i} / i=1,2, \ldots, m\right\}, V\left(P_{n}\right)=\left\{v_{j} \mid j=1,2, \ldots, n\right\}$ and $V(G)=\left\{v_{j} / j=1,2, \ldots, p\right\}$. Since

$$
\alpha^{\prime}\left(F_{m, n}\right)= \begin{cases}m, & m \geq n \\ m+\left\lfloor\frac{n}{2}\right\rfloor, & m<n\end{cases}
$$


Assume that the maximum matching set of $F_{m, n}$ and $G$ be

$$
M_{1}= \begin{cases}\left\{u_{i} u_{j} \mid i=j=1,2,3, \ldots, n\right\}, & m \geq n \\ \left\{u_{i} u_{j} \mid i=j=1,2,3, \ldots, m\right\} \cup & \\ \left\{v_{m+1} v_{m+2}, v_{m+3} v_{m+4}, \ldots, v_{m+2\left\lfloor\frac{n-m}{2}\right\rfloor+1} v_{m+2\left\lfloor\frac{n-m}{2}\right\rfloor}\right\}, & m<n ;\end{cases}
$$

$M_{2}=\left\{v_{j} v_{j+1} \mid j=1,3, \ldots, 2 k-1\right\}$,respectively.

case1 $m=n$. By lemma 2.3 we have $\alpha^{\prime}\left(H_{i j}\right)=\alpha^{\prime}\left(H_{i(i+1)}\right)=2 \alpha^{\prime}(G)$. Let $M_{i}^{*}=\left\{\left(u_{i}, w_{l}\right)\left(v_{j}, w_{k}\right) \mid i=j\right.$ and $\left.w_{l}, w_{k} \in M_{2}\right\}$. Therefore a matched in $F_{m, n} \otimes G$ is $\bigcup_{i=1}^{m} M_{i}^{*}$.

Hence $\alpha^{\prime}\left(F_{m, n} \otimes G\right) \geq\left|\bigcup_{i=1}^{m} M_{i}^{*}\right|=2 n \alpha^{\prime}(G)$.

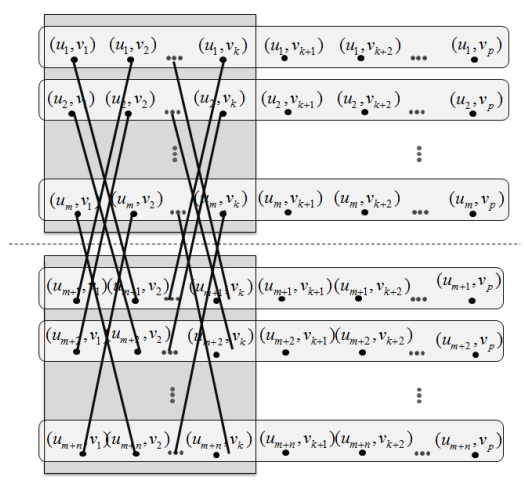

Figure 2: The case of $m=n$

Suppose that $\alpha^{\prime}\left(F_{m, n} \otimes G\right) \geq\left|\bigcup_{i=1}^{m} M_{i}^{*}\right|=2 n \alpha^{\prime}(G)$. Then there exist least two vertices $\left(u_{i}, w_{j}\right),\left(u_{j}, w_{i}\right) \in F_{m, n} \otimes G$ with edge $\left(u_{i}, w_{j}\right),\left(u_{j}, w_{i}\right) \notin M_{i}^{*}$. But is not possible.

Hence $\alpha^{\prime}\left(F_{m, n} \otimes G\right)=2 n \alpha^{\prime}(G)$ where $m=n$.

$\underline{\text { case2 }} m>n$. We have the matching $\bigcup_{i=1}^{m} M_{i}^{*}$. By definition of matching, we get another matching in $G$ be set of edges $M^{* *}=\{u v \in E(G) \mid u$ is not matched in maximum matching in $G$ and $\left.v \in N_{G}(u)\right\}$. So $\bigcup_{i=1}^{m} M_{i}^{*} \cup(m-n) M^{* *}$. 
Hence $\alpha^{\prime}\left(F_{m, n} \otimes G\right) \geq\left|\bigcup_{i=1}^{m} M_{i}^{*}\right|+(m-n)\left|M^{* *}\right|=2 n \alpha^{\prime}(G)+(m-n)\left|M^{* *}\right|$.

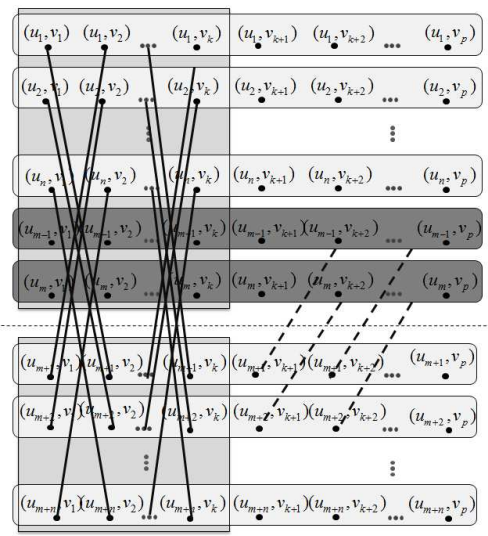

Figure 3: The case of $m>n$

Suppose that $\alpha^{\prime}\left(F_{m, n} \otimes G\right)>\left|\bigcup_{i=1}^{m} M_{i}^{*}\right|+(m-n)\left|M^{* *}\right|=2 n \alpha^{\prime}(G)+(m-$ $n)\left|M^{* *}\right|$, then there exist a matching $M$ is augmenting path. That is not true because each edges in $F_{m, n} \otimes G$ incident with edge in $M$.

Hence $\alpha^{\prime}\left(F_{m, n} \otimes G\right)=2 n \alpha^{\prime}(G)+(m-n)\left|M^{* *}\right|$, where $m>n$.

$\underline{\text { case } 3} m>n$ and $n-m=1$. We have the matching $\bigcup_{i=1}^{m} M_{i}^{*}$. By definition of matching,we get another matching in $G$ be set of edges $M^{* *}=\underset{m}{\{u v} \in E(G) \mid u$ is not matched in maximum matching in $G$ and $\left.v \in N_{G}(u)\right\}$. So $\bigcup_{i=1}^{m} M_{i}^{*} \cup M^{* *}$. Hence $\alpha^{\prime}\left(F_{m, n} \otimes G\right) \geq\left|\bigcup_{i=1}^{m} M_{i}^{*}\right|+\left|M^{* *}\right|=2 m \alpha^{\prime}(G)+\left|M^{* *}\right|$.

Suppose that $\alpha^{\prime}\left(F_{m, n} \otimes G\right)>\left|\bigcup_{i=1}^{m} M_{i}^{*}\right|+\left|M^{* *}\right|=2 m \alpha^{\prime}(G)+\left|M^{* *}\right|$, then there exist a matching $M$ is augmenting path. That is not true because each edges in $F_{m, n} \otimes G$ incident with edge in $M$.

Hence $\alpha^{\prime}\left(F_{m, n} \otimes G\right)=2 m \alpha^{\prime}(G)+\left|M^{* *}\right|$, where $m>n$ and $n-m=1$.

$\underline{\text { case } 4} m>n$ and $n-m$ is even. We have the matching $\bigcup_{i=1}^{m} M_{i}^{*} \cup(n-m) \alpha^{\prime}(G)$. 


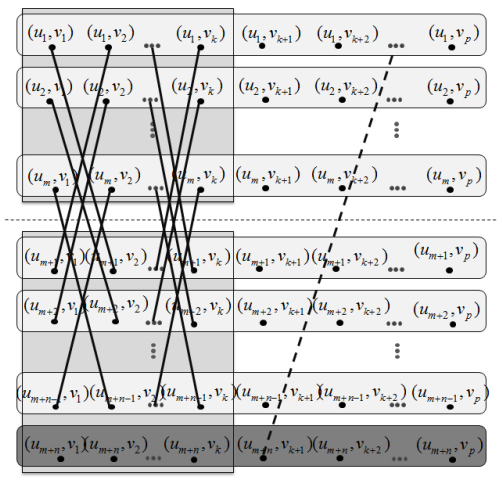

Figure 4: The case of $m>n$ and $n-m=1$

Hence $\alpha^{\prime}\left(F_{m, n} \otimes G\right) \geq\left|\bigcup_{i=1}^{m} M_{i}^{*}\right|+(n-m) \alpha^{\prime}(G)=2 m \alpha^{\prime}(G)+(n-m) \alpha^{\prime}(G)=$
$(m+n) \alpha^{\prime}(G)$

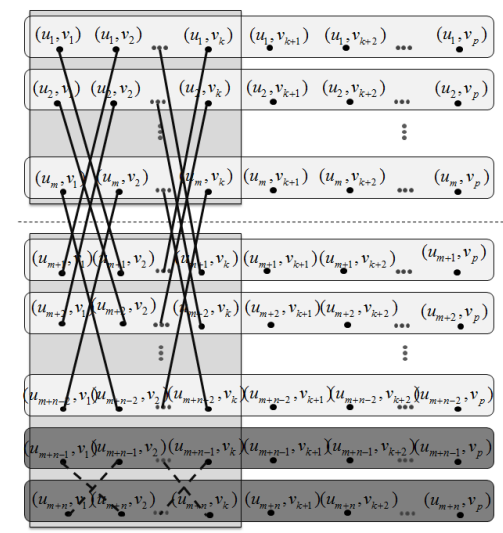

Figure 5: The case of $m>n$ and $n-m$ is even

Suppose that $\alpha^{\prime}\left(F_{m, n} \otimes G\right)>\left|\bigcup_{i=1}^{m} M_{i}^{*}\right|+(n-m) \alpha^{\prime}(G)=2 m \alpha^{\prime}(G)+(n-$ $m) \alpha^{\prime}(G)=(m+n) \alpha^{\prime}(G)$, then there exist a matching $M$ is augmenting path. That is not true because each edges in $F_{m, n} \otimes G$ incident with edge in $M$.

Hence $\alpha^{\prime}\left(F_{m, n} \otimes G\right)=2 m \alpha^{\prime}(G)+(n-m) \alpha^{\prime}(G)=(m+n) \alpha^{\prime}(G)$, where $m>n$ and $n-m$ is even. 
case5 $m>n$ and $n-m$ is odd. We have the matching $\bigcup_{i=1}^{m} M_{i}^{*} \cup(n-m-$ 1) $\alpha^{\prime}(G) \cup M^{* *}$, when $M^{* *}=\{u v \in E(G) \mid u$ is not matched in maximum matching in $G$ and $\left.v \in N_{G}(u)\right\}$.

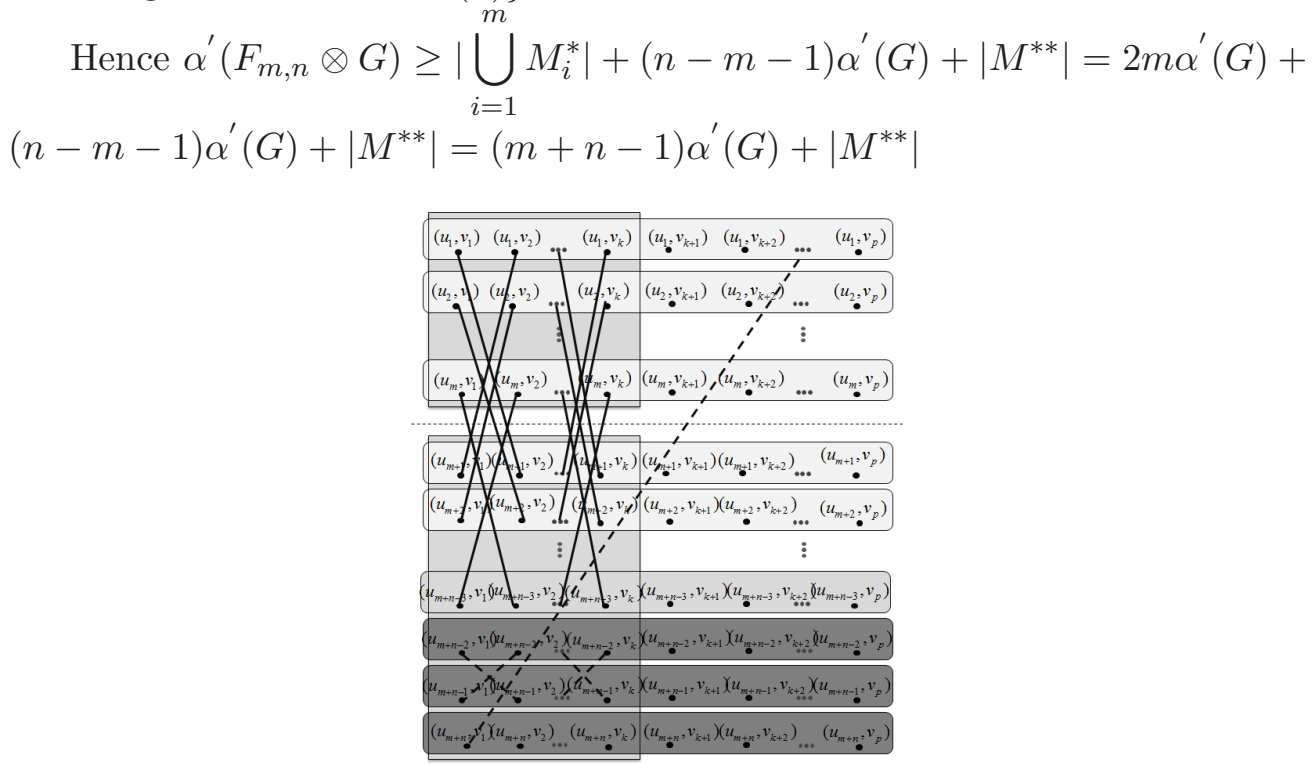

Figure 6: The case of $m>n$ and $n-m$ is odd

Suppose that $\left|\bigcup_{i=1}^{m} M_{i}^{*}\right|+(n-m-1) \alpha^{\prime}(G)+\left|M^{* *}\right| \geq 2 m \alpha^{\prime}(G)+(n-m-$ 1) $\alpha^{\prime}(G)+\left|M^{* *}\right|=(m+n-1) \alpha^{\prime}(G)+\left|M^{* *}\right|$, then there exist a matching $M$ is augmenting path. That is not true because each edges in $F_{m, n} \otimes G$ incident with edge in $M$.

Hence $\alpha^{\prime}\left(F_{m, n} \otimes G\right)=\left|\bigcup_{i=1}^{m} M_{i}^{*}\right|+(n-m-1) \alpha^{\prime}(G)+\left|M^{* *}\right|=(m+n-$ 1) $\alpha^{\prime}(G)+\left|M^{* *}\right|$, where $m>n$ and $n-m$ is odd.

\section{Edge Covering Number of the Graph of $F_{m, n} \otimes G$}

We begin this section by giving Lemma 3.1 that shows a relation of Matching number and Edge covering number. 
Lemma $3.1[2]$. Let $G$ be a simple graph with order $n$. Then $\alpha(G)+\beta(G)=$ $n$.

Next, we establish theorem 3.2 for a minimum Edge covering number of $F_{m, n} \otimes G$.

Theorem 3.2 Let $G$ be connected graph order $p$, then

$$
\beta^{\prime}\left(F_{m, n} \otimes G\right)= \begin{cases}2 n \beta^{\prime}(G), & m=n \text { or } m<n \text { and } \\ 2 n \beta^{\prime}(G)+(m-n)\left(p-\left|M^{* *}\right|\right), & m>n \text { is perfect matching } \\ 2 m \beta^{\prime}(G)+(n-m) p-\left|M^{* *}\right|, & m<n \text { and } n-m=1 \\ (m+n) \beta^{\prime}(G), & m<n \text { and } n-m \text { is even } \\ (m+n-1) \beta^{\prime}(G)-\left|M^{* *}\right|, & m<n \text { and } n-m \text { is odd, }\end{cases}
$$

where $M^{* *}=\{u v \in E(G) \mid u$ is not matched in maximum matching in $G$ and $\left.v \in N_{G}(u)\right\}$.

Proof. By theorem 2.3 and lemma 3.1, we can also show that $\alpha^{\prime}\left(F_{m, n} \otimes\right.$ $G)+\beta^{\prime}\left(F_{m, n} \otimes G\right)=(m+n) p$.

case $1 m=n$

$$
\begin{aligned}
\beta^{\prime}\left(F_{m, n} \otimes G\right) & =(m+n) p-2 n \alpha^{\prime}(G) \\
& =(n+n) p-2 n \alpha^{\prime}(G) \\
& =2 n p-2 n \alpha^{\prime}(G) \\
& =2 n\left(p-\alpha^{\prime}(G)\right) \\
& =2 n \beta^{\prime}(G)
\end{aligned}
$$

Hence $\beta^{\prime}\left(F_{m, n} \otimes G\right)=2 n \beta^{\prime}(G)$, where $m=n$.

case $2 m>n$

$$
\begin{aligned}
\beta^{\prime}\left(F_{m, n} \otimes G\right) & =(m+n) p-2 n \alpha^{\prime}(G)-(m-n)\left|M^{* *}\right| \\
& =((m-n)+2 n) p-2 n \alpha^{\prime}(G)-(m-n)\left|M^{* *}\right| \\
& =(m-n) p+2 n p-2 n \alpha^{\prime}(G)-(m-n)\left|M^{* *}\right| \\
& =2 n\left(p-\alpha^{\prime}(G)\right)+(m-n)\left(p-\left|M^{* *}\right|\right) \\
& =2 n\left(p-\beta^{\prime}(G)\right)+(m-n)\left(p-\left|M^{* *}\right|\right)
\end{aligned}
$$

Hence $\beta^{\prime}\left(F_{m, n} \otimes G\right)=2 n\left(p-\beta^{\prime}(G)\right)+(m-n)\left(p-\left|M^{* *}\right|\right)$, where $m>n$. 
$\underline{\text { case } 3} m>n$ and $n-m=1$

$$
\begin{aligned}
\beta^{\prime}\left(F_{m, n} \otimes G\right) & =(m+n) p-2 m \alpha^{\prime}(G)-\left|M^{* *}\right| \\
& =((-m+n)+2 m) p-2 m \alpha^{\prime}(G)-\left|M^{* *}\right| \\
& =(n-m) p+2 m p-2 m \alpha^{\prime}(G)-\left|M^{* *}\right| \\
& =2 m\left(p-\alpha^{\prime}(G)\right)+(n-m) p-\left|M^{* *}\right| \\
& \left.=2 m \beta^{\prime}(G)\right)+(n-m) p-\left|M^{* *}\right|
\end{aligned}
$$

Hence $\left.\beta^{\prime}\left(F_{m, n} \otimes G\right)=2 m \beta^{\prime}(G)\right)+(n-m) p-\left|M^{* *}\right|$, where $m>n$ and $n-m=1$. case $4 m>n$ and $n-m$ is even

$$
\begin{aligned}
\beta^{\prime}\left(F_{m, n} \otimes G\right) & =(m+n) p-(m+n) \alpha^{\prime}(G) \\
& =(m+n)\left(p-\alpha^{\prime}(G)\right) \\
& =(m+n) \beta^{\prime}(G)
\end{aligned}
$$

Hence $\beta^{\prime}\left(F_{m, n} \otimes G\right)=(m+n) \beta^{\prime}(G)$, where $m>n$ and $n-m$ is even.

case $5 m>n$ and $n-m$ is odd

$$
\begin{aligned}
\beta^{\prime}\left(F_{m, n} \otimes G\right) & =(m+n) p-(m+n-1) \alpha^{\prime}(G)-\left|M^{* *}\right| \\
& =(m+n) p-(m+n) \alpha^{\prime}(G)+\alpha^{\prime}(G)-\left|M^{* *}\right| \\
& =(m+n)\left(p-\alpha^{\prime}(G)\right)+p-\beta^{\prime}(G)-\left|M^{* *}\right| \\
& =(m+n) \beta^{\prime}(G)-\beta^{\prime}(G)+p-\left|M^{* *}\right| \\
& =(m+n-1) \beta^{\prime}(G)+p-\left|M^{* *}\right|
\end{aligned}
$$

Hence $\beta^{\prime}\left(F_{m, n} \otimes G\right)=(m+n-1) \beta^{\prime}(G)+p-\left|M^{* *}\right|$, where $m>n$ and $n-m$ is odd.

\section{References}

[1] Z.A. Bottreou, Y. Metivier, Some remarks on Kronecker product of graph, Inform. Process. Lett., 8 (1998), 279-286.

[2] D.B. West, Introduction to Graph Theory, Prentice-Hall (2001).

[3] P.M. Weichsel, The Kronecker product of graphs, Proc. Amer. Math. Soc., 8 (1962), 47-52. 